\title{
Effect of concentrated Kurozu, a traditional Japanese vinegar, on expression of hepatic miR-34a, -149-3p, and -181a-5p in high-fat diet-fed mice
}

\section{Yoshihiko Shibayama ${ }^{1}$, Masanobu Nagano ${ }^{2}$, Akira Fujii ${ }^{2}$, Kazunori Hashiguchi², Shunpei Morita1, Yoshitada Kubo' ${ }^{1}$, and Tsutomu Nakagawa ${ }^{1}$}

${ }^{1}$ Department of Drug Formulation, Faculty of Pharmaceutical Sciences, Health Sciences University of Hokkaido, 1757 Kanazawa, Tobetsu-cho, Ishikari-gun, Hokkaido 061-0293, Japan; ${ }^{2}$ Sakamoto Kurozu, Inc., 21-15, Uenosono-cho, Kagoshima 890-0052, Japan

Corresponding author: Yoshihiko Shibayama, Ph.D., Department of Drug Formulation, Faculty of Pharmaceutical Sciences, Health Sciences University of Hokkaido, 1757 Kanazawa, Tobetsu-cho, Ishikari-gun, Hokkaido 061-0293, Japan

Submission Date: October 8, 2019 Acceptance Date: December 26 ${ }^{\text {th }}$, 2019 Publication Date: January $31^{\text {st }}, 2020$

Citation: Shibayama Y, Nagano M, Fujii A, Hashiguchi K, Morita S, Kubo Y, Nakagawa T. Effect of concentrated Kurozu, a traditional Japanese vinegar, on expression of hepatic miR-34a, -149-3p, and -181a-5p in high-fat diet-fed mice. Functional Foods in Health and Disease 2020; 10(1): 1-17. DOI: https:/doi.org/10.31989/ffhd.v10i1.668

\begin{abstract}
Background: Long-term high-fat diet (HFD) feeding, which can induce obesity, can also induce nonalcoholic steatohepatitis (NASH) and liver tumorigenesis. A previous study reported that concentrated Kurozu (CK) supplementation reduced the incidence of HFD-induced hepatic steatosis in mice. It was showed that CK supplementation improved dyslipidemia in animal and clinical study. Small noncoding RNAs, micro RNAs (miRs), play crucial roles in the biology of cell functions, lipid metabolism and neoplasms. However, the effect of CK treatment on the relationship between HFD and expression of miRs is unclear.
\end{abstract}

Objective: To evaluate changes in the expression of hepatic miRs and lipid metabolismassociated genes on administering a HFD for 60 weeks in C57BL6J mice. The onset of hepatic steatosis induced by HFD treatment was also observed. 
Methods: The mice received a HFD, HFD with CK, or standard diet (SD) for 60 consecutive weeks. The effect of CK treatment on the expression levels of lipid metabolism-associated genes in the liver was evaluated.

Results: HFD feeding significantly increased expression of Tnf, and significantly decreased Adipoq and Mlxipl in the liver. The ingestion of CK elevated the expression levels of $P g c-1 \alpha$ and Igfbpl in the liver compared with the SD group. HFD feeding significantly increased the expression of $m i R-488-5 p$, and significantly decreased $m i R-29 b$ and $-122 a-5 p$ in the liver. The ingestion of CK elevated the expression levels of $m i R-34 a,-149-3 p$, and $-181 a-5 p$ in the liver compared with the SD group. Expression levels of $m i R-488-3 p$ in the serum HFD group were significantly higher than in the SD group. The ingestion of CK elevated the expression levels of $m i R-181 a-5 p$ in the serum compared with the SD group.

Conclusion: These results suggest that CK supplementation reduced the onset of hepatic hyperplasia, and increased hepatic miR-34a, -149-3p, and -181a-5p. These miRs may function as suppressors of tumors caused by HFD feeding.

Key Words: High-fat diet, carcinogenesis, Kurozu, microRNA, miR-34a, miR-122a-5p, miR-149, miR-181a, miR-488

\section{BACKGROUND:}

Black unpolished rice vinegar, Kurozu, is a traditional vinegar with a dark amber color. Kurozu is a widely used seasoning, and it has been commonly used as a healthcare supplement. Previous studies reported that concentrated Kurozu (CK) reduced the onset of hepatic steatosis following high-fat diet ingestion [1], improved the symptoms of hypertension, allergies, and hypercholesterolemia [2], enhanced carbohydrate metabolism [3], and inhibited tumor growth [4-6]. Tanizawa $\mathrm{H}$ et al. reported that Kurozu showed effects of decreasing serum cholesterol levels in both normal and high cholesterol diet-fed mice [7]. In a case study, the extract of Kurozu treatment showed significant decrease of total cholesterol in patients with cardiovascular disease [8]. Hamadate $\mathrm{N}$ et al. reported that concentrated Kurozu supplementation significantly decreased body weight and nearly significant decreased values of body mass index in healthy adults with obesity, and decreased hip circumference and fat-derived energy consumption increased while suppressing carbohydrate-derived energy consumption in obesity healthy subjects [9]. Abe A et al. reported that Kurozu decreased visceral fat accumulation in obese healthy subjects [10]. These reports suggest CK supplementation has significant effect on dyslipidemia. 
Excessive and long-term intake of alcohol is a representative cause of fatty liver. Non-alcoholic fatty liver disease (NAFLD) is a hepatic manifestation of metabolic syndrome that develops regardless of the level of alcohol intake. NAFLD is accompanied by obesity, diabetes, high blood pressure, and dyslipidemia [11-13]. NAFLD often considered a relatively benign condition, will progress to more severe stages of liver disease including NASH (non-alcoholic steatohepatitis) with or without fibrosis. NAFLD is a major etiology of hepatocellular carcinoma, based on the rising prevalence of obesity [14]. Oxidative stress promotes hepatocyte cell death and activation of inflammatory pathways, including expression of the pro-inflammatory cytokine tumor necrosis factor (TNF), which leads to advanced fibrosis and cirrhosis $[15,16]$.

MicroRNAs (miRs) are short non-coding RNAs involved in post-transcriptional regulation of genes. miRs can bind to bind to complementary target sites in mRNA genes and can cause translation repression, cleavage, deadenylation, or degradation of target mRNA genes [17-19]. Recently, miRs have been reported to be important regulatory players in a variety of biological processes, including complex metabolic processes, such as energy and lipid metabolism in the context of diabetes and obesity. Mitochondrial function has been firmly linked to obesity; for example, mitochondrial dysfunction leads to a decrease in substrate oxidation, particularly fatty acids, resulting in lipid accumulation. miRs have been recognized as regulators of mitochondrial function [20]. Accumulating evidence indicates that miRs also play an integral role in adipose tissue formation and function [21]. It was reported that alteration of $\mathrm{miR}$ expression in response to genetic/epigenetic factors or environmental conditions may contribute to steatosis onset and NAFLD progression [22, 23]. miRs have been identified in serum, and plasma and are stable in the circulation. miRs are preferentially packaged and released in exosomes. Exosomes are small vesicles (30-90 nm) that are released from many cellular types into extracellular spaces. miRs in serum are emerging as non-invasive diagnostic and prognostic biomarkers [22, 24].

The miRs affect cancer biology, including cell proliferation, adhesion, invasion, and apoptosis [25]. miR-34a is recognized as a tumor suppressor and its ability to regulate the expression of multiple targets has been implicated in tumorigenesis and cancer progression [26]. miR-149 also has a potential role in cancer proliferation, apoptosis, migration, and invasion [27]. miR-29b, 122a, 143, 181a, 488-5p, and 543-2 also play potential roles in the biology of cancer and lipid metabolism.

In the present study, the effect of CK supplementation on miR expression was evaluated in mice fed a high-fat diet. The effect of miR expression on tumorigenesis in high-fat diet-fed mice was also evaluated. 


\section{METHODS:}

\section{Reagents}

Concentrated Kurozu (CK) from a jar (10-fold concentration) was obtained from Sakamoto Kurozu, Inc. (Kagoshima, Japan). CK contained $1.73 \%$ lactic acid, $0.01 \%$ acetic acid, and $17.4 \%$ of solid contents. PCR primers were obtained from Fasmac Co., Ltd. (Atsugi, Japan). The PCR primers are shown in Table 1. The real-time PCR master mix, THUNDERBIRD SYBR qPCR Mix and reverse transcriptase, ReverTra Ace, were purchased from TOYOBO Co., Ltd. (Osaka, Japan). The miScript Primer Assays and miScript II RT Kit were purchased from Qiagen (Valencia, CA, USA). All other reagents were purchased from Wako Pure Chemical Industries (Osaka, Japan).

\section{Diets}

A standard-diet (SD) feed, CE-2, and high-fat diet (HFD) feed $\left(\right.$ QuickFat ${ }^{\circledR}$ ) were purchased from CLEA Japan, Inc. (Tokyo, Japan). CE-2 contains $3.40 \mathrm{kcal} / \mathrm{g}$, and $4.8 \%$ fat and was made from soy beans and wheat germ. QuickFat contains $4.06 \mathrm{kcal} / \mathrm{g}$, and $13.9 \%$ fat and was made from cattle suet and wheat germ. The HFD with CK diet contained a concentration of $0.6 \% \mathrm{CK}(\mathrm{W} / \mathrm{W}$ ) according to a previous report. The concentration of Kurozu in feed was configured to a 1-day average amount of food ingested by a mouse and the standard intake volume of Kurozu for humans as $30 \mathrm{~mL}$ per day. The ingested amount of Kurozu forage per mouse per day corresponded to the average intake in humans per body surface area. $[1,28]$.

\section{Animals}

Male C57BL6J mice (10 weeks old, Hokudo, Sapporo, Japan) were housed under standard conditions $\left(21 \pm 2^{\circ} \mathrm{C}\right.$, ventilated room, 12-h light/dark cycle). The chronic treatment for 60 weeks was performed as follows: the animals were given free access to food and water during the experimental period; the SD group was given CE-2, the HFD group was given QuickFat, and the CK group was given CK-containing QuickFat. The calorie intake was calculated based on the decrease in the weight of the feed once a week. Mice were terminated by exsanguination after blood collection. The target tissues were promptly removed and stored to stabilize and protect cellular RNA in RNAlater at $-30^{\circ} \mathrm{C}$ until RT-PCR analysis. Serum prepared from each mouse was stored at $4{ }^{\circ} \mathrm{C}$ until analysis. Hemolysate samples were excluded from samples for analysis. The present study was approved (approved number: 026) based on the criteria of Health Sciences University of Hokkaido for the use and care of experimental animals. The number of mice was calculated to observe significant difference under condition was at the 0.05 significance level $(\alpha)$, and at the 0.8 statistical power $(\beta)$ according to the previous result [1]. 


\section{Assay and histological analysis}

Total RNA was isolated from target tissues using ISOGEN Reagent according to the manufacturer's instructions. Single-stranded cDNA was synthesized by reverse-transcriptase (RT) using ReverTra Ace or miScript II RT Kit based on the manufacturer's instructions. Real-time PCR was performed according to a previous report [1]. Beta-2-microglobulin $(B 2 m)$ was analyzed as a reference gene for mRNA. MiR-16 was analyzed as a reference gene for miR [29, 30]. Biochemical analysis was performed by Oriental Yeast Co., Ltd. (Tokyo, Japan). The liver was fixed with $10 \%$ formalin neutral buffer solution. Paraffin sections were stained with hematoxylin-eosin by conventional methods.

Table 1. The sequences of primers

\begin{tabular}{lll}
\hline Gene & Sense $\left(5^{\prime}-3^{\prime}\right)$ & Anti $\left(5^{\prime}-3^{\prime}\right)$ \\
\hline$B 2 m$ & GACCGGCCTGTATGCTATCC & TCAGTCTCAGTGGGGGTGAA \\
Tnf & GATCGGTCCCCAAAGGGATG & ACAAGGTACAACCCATCGGC \\
Sirt1 & CCTTTCAGAACCACCAAAGCG & GGCGAGCATAGATACCGTCT \\
Pgc-1 $\alpha$ & AGCGGTTCTCACAGAGACAC & GGTTTGGTGTGAGGAGGGTC \\
Igfbpl & TCTGAGAGCCCAGAGATGACA & GTAGACACACCAGCAGAGTCC \\
Lpin1 & GGAAAAGGAGGAGGAAGGCC & TACGATGCTGACTGGGGGTA \\
Adipoq & ATGGCAGAGATGGCACTCCT & GCCAGTGCTGCCGTCATAAT \\
Mlxipl & CACTCACCCACCTCTTCGAGA & TCCCGGCATAGCAACTTGAGC \\
\hline
\end{tabular}

\section{RESULTS:}

Chronic treatment with Kurozu reduced the incidence of hepatic steatosis in high-fat diet-fed mice

Ten-week-old C57BL6J mice were fed HFD containing CK for 60 weeks. The liver and blood were collected at 70 postnatal weeks. The HFD treatment significantly increased body weight compared with the SD group (Figure 1). Calorie intakes of the HFD with CK group was significantly higher than in the SD or HFD group (SD group: $10.09 \pm 0.48 \mathrm{kcal} /$ day, HFD group: $11.36 \pm 0.25 \mathrm{kcal} /$ day, HFD with $\mathrm{CK}$ group: $12.21 \pm 0.37 \mathrm{kcal} /$ day, standard error mean \pm standard error. $p<0.001$, compared with SD group, $p<0.01$, compared with HFD group). Supplementation of CK significantly decreased body weight compared with the HFD group during postnatal weeks 15-19. Body weight of the HFD with CK group was higher than that of the HFD group, but significant differences were not observed. HFD feeding significantly elevated total cholesterol (T-CHO), low-density lipoprotein cholesterol (LDL-C), and high-density lipoprotein cholesterol (HDL-C) in the serum. No significant differences were noted in the values of alanine aminotransferase (ALT) or alkaline phosphatase (ALP) among the 3 groups. Supplementation of CK significantly increased the liver weight compared with the HFD group (Figure 2). HFD feeding significantly increased the incidence 
of hepatic steatosis based on comparison among the 3 groups ( $p=0.021$, Figure 2). Supplementation of CK suppressed the incidence of hepatic steatosis on HFD feeding (Figures 2 and 3).

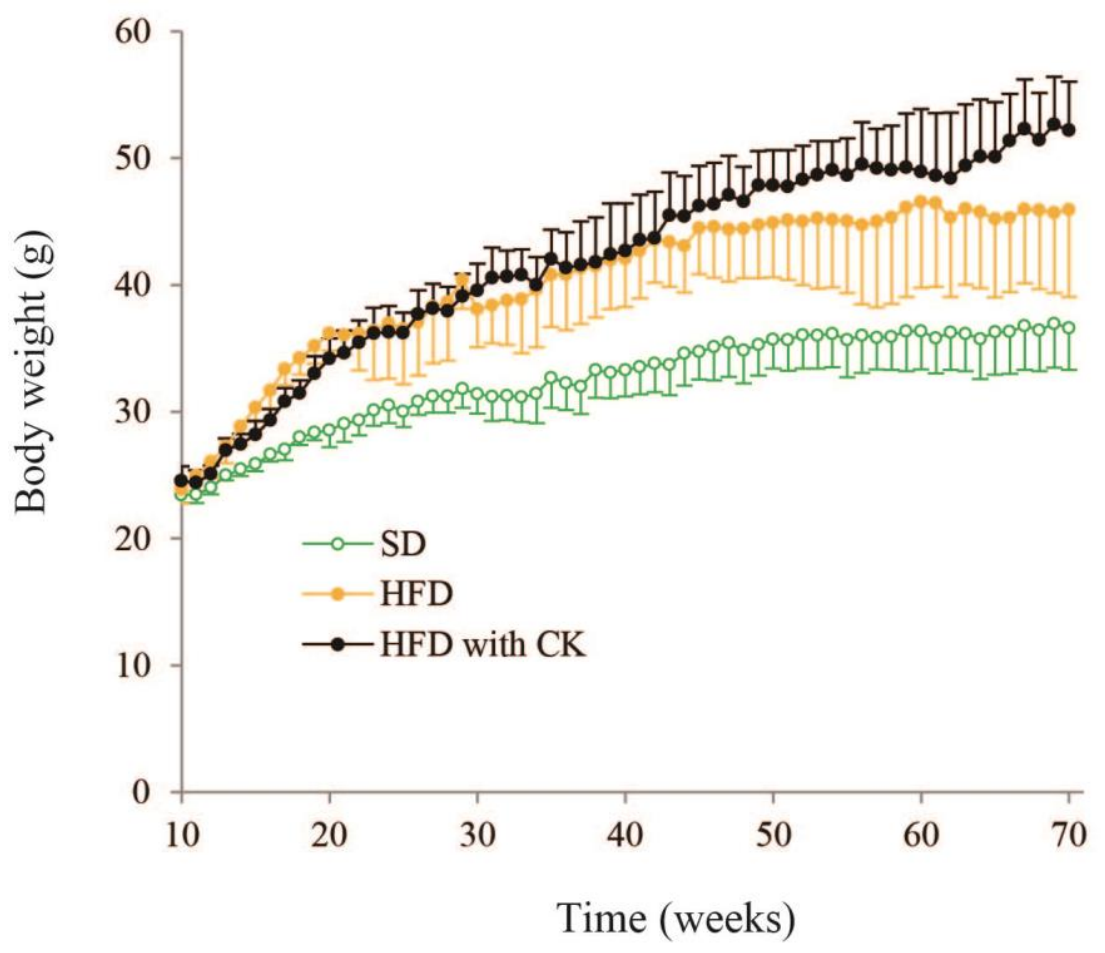

Figure 1. Body weight changes. The average weekly values of each group are shown. C57BL6J mice fed SD, HFD, or HFD with CK forage for 60 consecutive weeks. The body weight of HFD with CK group was significantly lower than in the HFD group during postnatal weeks 15-19. Statistical analysis was performed using the Tukey-Kramer test.
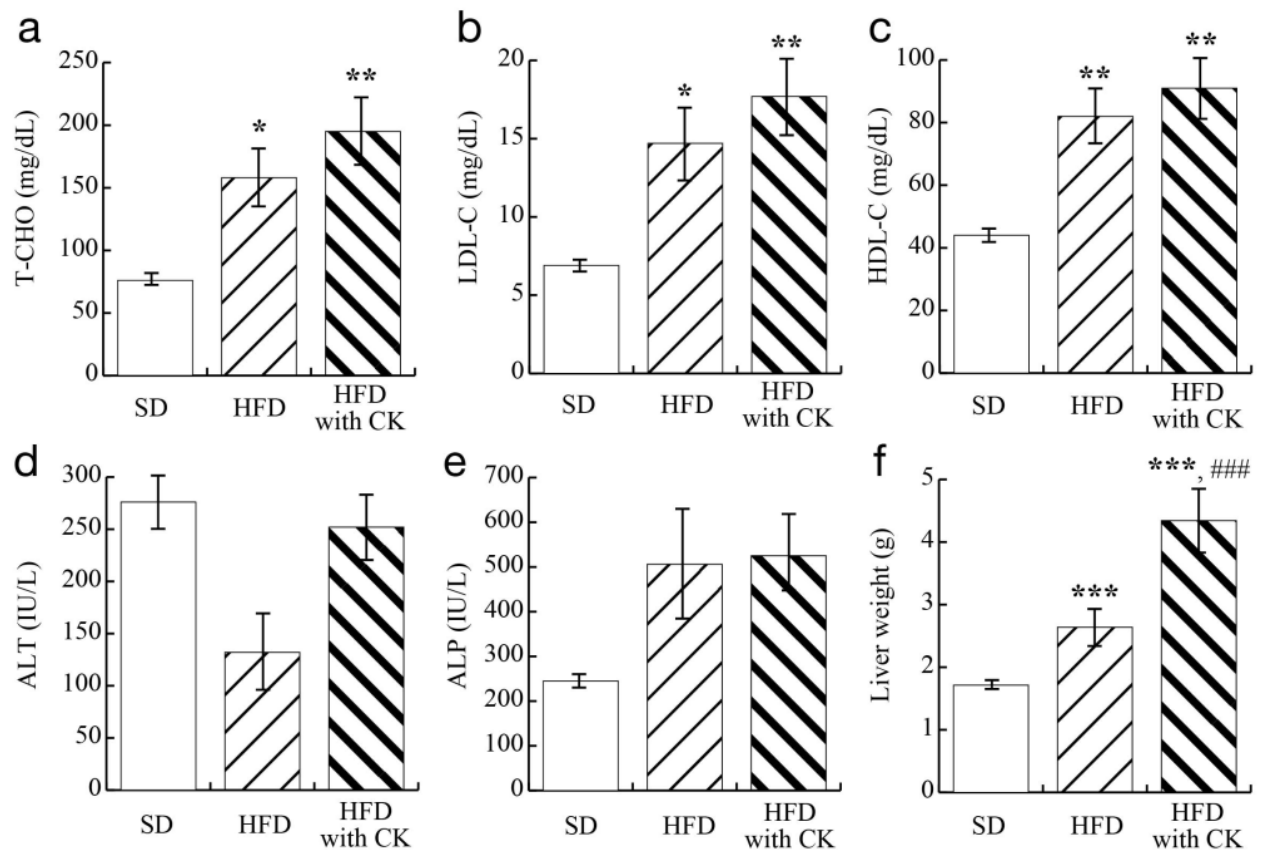

Figure 2. Biochemical data on serum and liver weights. Each value is the mean \pm standard error. *: $p<0.05, * *: p<0.01, * * *: p<0.001$, significantly different from the SD group, \#\#\#: $p<0.001$, significantly different from 
the HFD group, Tukey-Kramer test. a, T-CHO: total cholesterol; b, LDL-C: low-density lipoprotein cholesterol; c, HDL-C: high-density lipoprotein cholesterol; d, ALT: alanine aminotransferase; e, ALP: alkaline phosphatase; f, whole liver weight, CK supplementation significantly increased the liver weight compared with the SD or HFD group.

\section{CK treatment altered expression levels of metabolism-related genes in the liver}

To evaluate the effects of CK treatment on expression levels of metabolism-related genes. Expression levels of mRNA and miR were evaluated by real-time PCR analysis. HFD treatment significantly increased the mRNA expression levels of Tnf, but decreased Adipoq and Mlxipl in the liver. CK supplementation significantly increased the mRNA expression levels of $P g c-1 \alpha$ and $I g f b p 1$ in the liver (Table 2).

HFD treatment significantly increased the miR expression levels of $m i R-488-5 p$, but decreased $m i R-29 b$ and $m i R-122 a-5 p$ in the liver. CK supplementation significantly increased the miR expression levels of $m i R-34 a, m i R-149-3 p$, and $m i R-181 a-5 p$ in the liver (Table 3 ). CK supplementation also increased the miR expression levels of miR-181a-5p in the serum (Table 4).
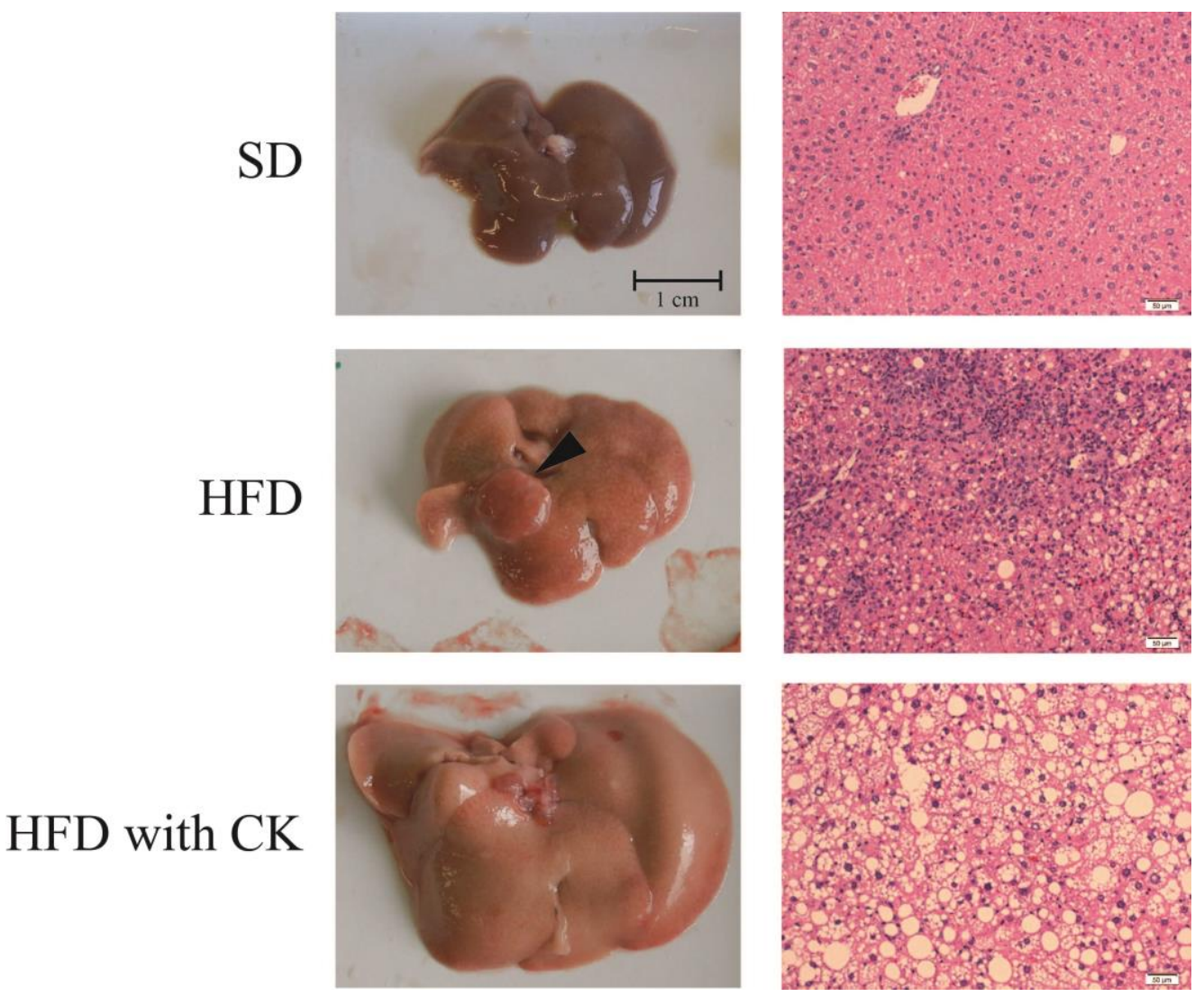

Figure 3. Microscopic images and gross morphology of the liver. Upper images show the SD group. Middle image shows hepatic steatosis in the HFD group. The liver shows hyperplasia. Liver hyperplasia was observed in five of seven mice in the HFD group ( $p=0.021)$. It was not observed in SD and HFD with CK groups. Statistical analyses were performed using Fisher's exact test. 
Table 2. Alterations of mRNA expression in the liver relating to lipid metabolism following ingestion of $\mathrm{CK}$ in mice

\begin{tabular}{llll}
\hline & SD & HFD & HFD with CK \\
\hline Tnf & $1.00 \pm 0.18$ & $30.25 \pm 8.70^{*}$ & $24.54 \pm 5.04^{*}$ \\
Sirt1 & $1.00 \pm 0.15$ & $0.64 \pm 0.11$ & $0.66 \pm 0.17$ \\
Pgc-1 & $1.00 \pm 0.33$ & $2.70 \pm 0.64$ & $5.95 \pm 1.93^{*}$ \\
Igfbpl & $1.00 \pm 0.24$ & $1.55 \pm 0.43$ & $2.67 \pm 0.47^{*}$ \\
Lpin1 & $1.00 \pm 0.24$ & $0.65 \pm 0.13$ & $0.41 \pm 0.17$ \\
Adipoq & $1.00 \pm 0.16$ & $0.05 \pm 0.02^{* * *}$ & $0.04 \pm 0.02^{* * *}$ \\
Mlxipl & $1.00 \pm 0.07$ & $0.58 \pm 0.05^{* *}$ & $0.46 \pm 0.07^{* * *}$ \\
\hline
\end{tabular}

C57BL6J mice received SD, HFD, or HFD with CK forage for 60 consecutive weeks. The values are ratios of target gene / B2m expression levels. Each value is the mean \pm standard error. Statistical analyses were performed using the Tukey-Kramer test. Significantly different from the SD group: *: $p<0.05, * *: p<0.01, * * *$ : $p<0.001$

Table 3. Alterations of miR expression in the liver related to lipid metabolism following ingestion of $\mathrm{CK}$ in mice

\begin{tabular}{llll}
\hline & SD & HFD & HFD with CK \\
\hline$m i R-29 b$ & $1.00 \pm 0.18$ & $0.22 \pm 0.02^{* *}$ & $0.46 \pm 0.10^{*}$ \\
$m i R-34 a-5 p$ & $1.00 \pm 0.16$ & $1.92 \pm 0.42$ & $5.01 \pm 0.80^{* *, \# \#}$ \\
$m i R-122 a-5 p$ & $1.00 \pm 0.08$ & $0.60 \pm 0.06^{* *}$ & $0.50 \pm 0.04^{* *}$ \\
$m i R-143$ & $1.00 \pm 0.16$ & $0.91 \pm 0.09$ & $1.09 \pm 0.31$ \\
$m i R-149-3 p$ & $1.00 \pm 0.15$ & $1.14 \pm 0.31$ & $23.76 \pm 9.35^{*, \#}$ \\
$m i R-181 a-5 p$ & $1.00 \pm 0.19$ & $1.24 \pm 0.20$ & $3.61 \pm 1.09^{*}, \#$ \\
$m i R-488-3 p$ & $1.00 \pm 0.11$ & $37.49 \pm 7.57 *$ & $43.08 \pm 12.43^{*}$ \\
$m i R-543-3 p$ & $1.00 \pm 0.20$ & $1.35 \pm 0.58$ & $1.35 \pm 0.57$ \\
\hline
\end{tabular}

C57BL6J mice received SD, HFD, or HFD with CK forage for 60 consecutive weeks. The values were calculated from the ratio of target gene / miR-16 expression levels. Each value is the mean \pm standard error. Statistical analyses were performed using the Tukey-Kramer test. Significantly different from the SD group: *: $p<0.05, * *: p<0.01$. Significantly different from the HFD group: \#: $p<0.05$. 
Table 4. Changes of miR expression in the serum related to lipid metabolism following ingestion of $\mathrm{CK}$ in mice

\begin{tabular}{llll}
\hline & SD & HFD & HFD with CK \\
\hline$m i R-29 b$ & $1.00 \pm 0.11$ & $0.52 \pm 0.11$ & $0.68 \pm 0.14$ \\
$m i R-34 a-5 p$ & $1.00 \pm 0.15$ & $1.02 \pm 0.25$ & $1.85 \pm 0.33$ \\
$m i R-122 a-5 p$ & $1.00 \pm 0.30$ & $1.55 \pm 0.41$ & $1.64 \pm 0.31$ \\
$m i R-143$ & $1.00 \pm 0.18$ & $0.44 \pm 0.16$ & $0.54 \pm 0.11$ \\
$m i R-149-3 p$ & $1.00 \pm 0.20$ & $0.94 \pm 0.26$ & $3.99 \pm 2.04$ \\
$m i R-181 a-5 p$ & $1.00 \pm 0.14$ & $0.89 \pm 0.24$ & $9.74 \pm 2.52^{* *}, \# \#$ \\
$m i R-488-3 p$ & $1.00 \pm 0.20$ & $21.21 \pm 4.59^{*}$ & $22.69 \pm 6.40^{*}$ \\
$m i R-543-3 p$ & $1.00 \pm 0.18$ & $0.84 \pm 0.18$ & $0.76 \pm 0.14$ \\
\hline
\end{tabular}

C57BL6J mice received SD, HFD, or HFD with CK forage for 60 consecutive weeks. The values were calculated from the ratio of target gene / miR-16 expression levels. Each value is the mean \pm standard error. Statistical analyses were performed using the Tukey-Kramer test. Significantly different from the SD group: *: $p<0.05, * *: p<0.01$. Significantly different from the HFD group: \#\#: $p<0.01$.

\section{DISCUSSION:}

In this study, mice were fed HFD with CK for 60 consecutive weeks to elucidate the contribution of miRs to the carcinogenicity of HFD feeding. HFD feeding significantly increased the body weight compared with the SD group. The body weight of the HFD with CK group was significantly lower than in the HFD group during postnatal weeks 15-19 (Figure 1). A previous study reported that CK supplementation significantly suppressed body weight in mice [1]. Hamadate N, et al. reported that powdery concentrated Kurozu treatment for 12 weeks decreased the body weight of humans [8]. In old age, body weights were higher in the CK group than in the HFD group. A previous study also reported that CK supplementation reduced body weights in older age [1]. CK supplementation significantly increased the liver weight compared with the SD or HFD group (Figure 2f). Tong LT, et al. reported that $\mathrm{CK}$ treatment decreases the adipocyte size and the number of cells in subcutaneous adipose tissues of a CK-treated group was significantly increased compared with a control group [31]. It was estimated that the difference in liver weight may affect the difference in body weight. HFD feeding significantly increased the cholesterol concentration but not ALT or AST in the serum (Figure 2). Fukuayma N et al. reported that concentrated Kurozu supplementation inhibited inflammation and oxidative stress of ulcerative colitis induced by dextran sulfate sodium treatment at a dose of $0.32 \%(\mathrm{w} / \mathrm{w})$ supplementation in forage [32]. Previous reports showed that CK supplementation improved cholesterol levels in 
mice, and showed no significant differences levels of AST, ALP and T-CHO in the clinical study $[7,9,11]$. It was estimated that CK supplementation did not show hepatic toxicity from CK supplementation.

HFD feeding significantly increased the onset of hepatic hyperplasia (Figure 3, middle images). Hyperplasia of the liver was only observed in the HFD group (number of site of hyperplasia / number of mice, HFD group, 5 / 7; HFD with CK group, 0 / 7; SD group, 0 / 7; $p=0.021$, Fisher's exact test.). A previous study reported that CK supplementation reduced the onset of hepatic steatosis at 110 postnatal weeks [1]. The effect of Kurozu to prevent carcinogenesis has been reported in rats [6] and cell lines in vitro $[6,33]$. The effect of CK to prevent carcinogenesis was also observed in this study.

HFD feeding altered metabolism-associated genes in the liver. HFD feeding significantly increased expression levels of $\operatorname{Tnf}(T N F-\alpha$, Tumor Necrosis Factor). It was reported that HFD increased expression levels of Tnf [34]. Many studies have provided evidence that TNF derived from inflammatory liver macrophages is indispensable for NASH and steatohepatitic HCC development under HFD feeding [35]. HFD feeding significantly increased expression levels of Adipoq (Adiponectin). It was reported that HFD treatment decreased expression levels of Adipoq [36]. The same effects of the alteration of gene expression were observed in this study. CK supplementation significantly increased expression levels of $P g c-1 \alpha$ and Igfbpl. A previous study reported the same effect [1]. Alteration of expression levels of Sirt1 and Lipin1 was not observed in this study. Mlxipl (MLX interacting protein like, also known as Chrebp) functions as a central metabolic coordinator in response to environmental and hormonal signals. Mlxipl forms a heterodimeric complex and binds and activates, in a glucose-dependent manner, carbohydrate response element motifs in the promoters of triglyceride synthesis genes [37]. In the liver in this study, Chronic treatment with a high-fat diet induced expression of Mlxipl. A previous study involving chronic treatment for 24 weeks demonstrated the same effect using microarray analysis (expression ratio: 0.13, Gene Expression Omnibus accession: GSE39549) [38].

The miR-29b is known to critically affect cancer progression by functioning as a tumor suppressor. miR-29b induces apoptosis and inhibits metastasis in human cancer cells [39]. A recent study reported that within the miR-29 family, miR-29a levels in serum of NAFLD patients were significantly lower than in healthy controls [40]. In this study, HFD feeding significantly suppressed the expression of hepatic miR-29b. The effect of CK supplementation did not influence on the expression of miR-29b alteration. miR-34a-5p (previous ID: miR-34a, accession MIMAT0000542) is recognized as a tumor suppressor, and its ability to regulate the expression of multiple targets (e.g., Myc, P53, CDK4/6) has been implicated in tumorigenesis and cancer progression [26]. A previous cohort study reported 
that miR-34a was associated with NAFLD severity [24]. CK supplementation increased expression levels of hepatic miR-34a, resulting in a reduced incidence of hepatic steatosis induced by HFD. Shimoji Y, et al. reported that Kurozu contained polyphenolic compounds, such as dihydroferulic and dihydrosinapic acids, which exhibit antioxidative activities [41]. Treatment with the bioactive polyphenol Honokiol increased expression of miR-34a in breast cancer cells [42]. Polyphenolic compounds in CK may act as activators of miR-34a, but the mechanism is unclear. miR-122 (previous ID: miR-122a, accession MIMAT0000246) plays critical roles in liver homeostasis and hepatocarcinogenesis [43]. miR-143 was identified as a positive regulator of human adipocyte differentiation. miR-143 expression was increased in the mesenteric adipose of HFD-fed mice [44]. HFD feeding decreased hepatic miR-143 expression, but alterations of $m i R-122 a-5 p$ and -143 expressions in the serum were not observed in this study. miR-149-3p (previous ID: miR-149*, accession MIMAT0016990) also has potential roles in cancer proliferation, apoptosis, migration, and invasion [23]. Shin $\mathrm{CH}$, et al. reported that miR-149-3p inhibited clonogenicity and induced apoptotic cell death via Polo-like kinase 1 inhibition [45]. miR-149-3p also plays crucial roles in cell proliferation, migration, and invasion by inhibiting Foxm1 and S100A4 [46, 47]. The miR-149-3p-upregulating effect of CK may influence the development of hepatic hyperplasia. Recent research verified that miR-181a-5p (previous ID: miR-181a, accession MIMAT0000210) could modulate cell apoptosis by targeting apoptosis-related genes, including the Bcl-2 family, P53, ATM, PRKCD, PBX3, and RALA [48]. CK supplementation increased expression of hepatic miR-181a-5p, and the inhibitory effect of miR-181a-5p may affect the development hepatic hyperplasia. In this study, HFD feeding increased the expression of $m i R-488-3 p$ (previous ID: miR-488, accession MIMAT0003450) in the liver. It is unclear whether HFD feeding affects miR-488 expression. Yu DL, et al. reported that Janus kinase 1 (JAK1) is a direct target of miR-488-3p [49]. Tang LY, et al. reported that JAK1/STAT pathways play critical roles in liver fibrosis by TGF- $\beta$ signaling [50]. miR-488-3p expression in the liver is altered by HFD treatment. Further investigations are needed to understand the role of miR-488-3p in the liver. miR-543-3p (previous ID: miR-543, accession MIMAT0003168) suppresses cell proliferation, migration, and invasion by targeting SIRT1 [51]. Mantilla-Escalante DC, et al. reported that miR-543-3p expression in serum was upregulated after high-fat diet intake in male mice [52]. Alteration of $m i R-543-5 p$ expression was not observed in this study. CK supplementation significantly elevated the expression levels of $m i R-181 a-5 p$ in the serum. The results suggest that expression levels of $m i R-181 a-5 p$ in the serum may be a biomarker of carcinogenicity in the presence of NAFLD.

The miR-34a, -149-3p and -181a-5p are key regulators of carcinogenesis and lipid metabolism, and the upregulating effects of $m i R-34 a,-149-3 p$, and $-181 a-5 p$ may result from 
CK supplementation. Taken together, our data demonstrate that upregulated expression of $m i R-34 a,-149-3 p$, and $-181 a-5 p$ may reduce the onset of hepatic hyperplasia. To illustrate the effects of CK on hepatic carcinogenesis, a possible signaling pathway is indicated in Figure 4.
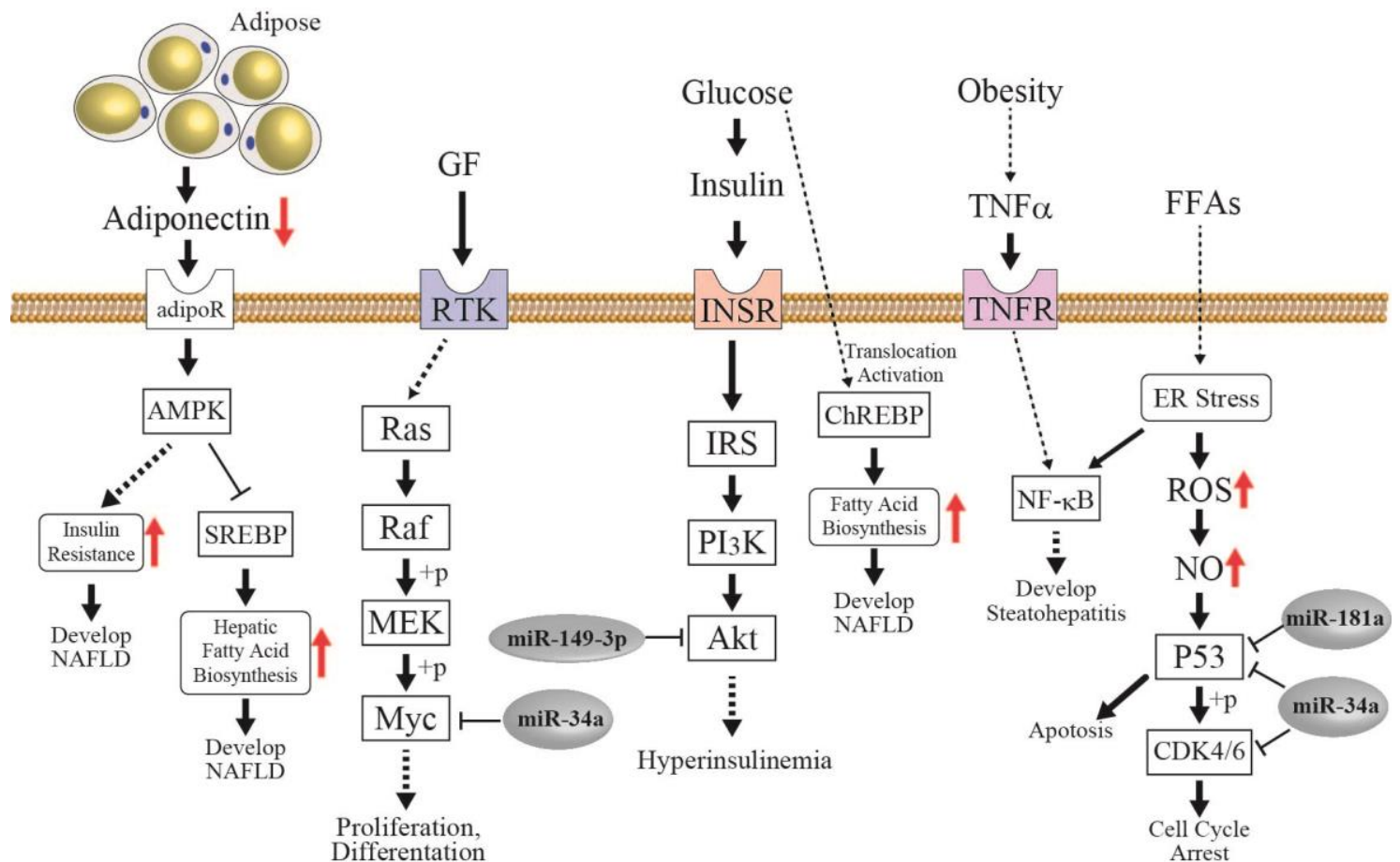

Figure 4. A possible mechanism of suppressing HFD-induced carcinogenesis by CK supplementation. The present study indicated that CK supplementation increased expression of hepatic $m i R-34 a,-149-3 p$, and $-181 a-5 p$. It was reported that miR-34a, -149-3p, and -181a-5p inhibited Myc, Akt, P53, and CDK4/6. The target genes of miRs play crucial roles in the development of neoplasms. Abbreviations: adipoR, adiponectin receptor; ChREBP, carbohydrate response element binding protein; ER, endoplasmic reticulum; FFAs, free fatty acids; GF, growth factor; INSR, insulin receptor; NO, nitric oxide; ROS, reactive oxygen species; RTK, receptor tyrosine kinase; SREBP, sterol regulatory element-binding protein; TNFR, TNF receptor.

\section{CONCLUSION:}

The results suggest that $\mathrm{CK}$ supplementation increases the expression levels of $T n f$, and decreases Adipoq and Mlxipl in the liver. HFD feeding also increases expression of $m i R-488-5 p$, and decreases $m i R-29 b$ and $-122 a-5 p$ in the liver. The ingestion of CK elevated the expression levels of $m i R-34 a,-149-3 p$, and $-181 a-5 p$ in the liver. CK supplementation elevated the expression levels of $m i R-181 a-5 p$ in the serum. The results suggest that expression levels of $m i R-181 a-5 p$ in the serum may be a biomarker of carcinogenicity in the presence of NAFLD. 
List of abbreviations: CK, Concentrated Kurozu; HFD, High-fat diet; Non-alcoholic steatohepatitis, miR, microRNA; NASH; Non-alcoholic fatty liver disease, NAFLD.

Competing interests: Masanobu Nagano, Akira Fujii, and Kazunori Hashiguchi are employees of Sakamoto Kurozu, Inc.

Authors' contributions: YS conducted the study. YS, SM, YK and TN performed animal experiments and analyses. MN, AF, and KH generated CK. YS prepared the manuscript, and all authors contributed to proofreading and revisions.

Acknowledgements and Funding: The authors thank Drs. Ken Iseki and Tekehiro Yamada (Hokkaido University) for their assistance of microscope imaging. This work was supported by grants from Sakamoto Kurozu, Inc.

\section{REFERENCES:}

1. Shibayama Y, Nagano M, Hashiguchi K, Fujii A, Iseki K: Supplementation of concentrated Kurozu, a Japanese black vinegar, reduces the onset of hepatic steatosis in mice fed with a high-fat diet. Funct Foods Health Dis 2019, 9:276-296.

2. Murooka Y, Yamashita M: Traditional healthful fermented products of Japan. J Ind Microbiol Biotechnol 2008, 35:791-798.

3. Nagano M, Fujii A, Aoyama Y, Kurita M, Fujii M: Effects of Kurozu Moromi Powder and Kurozu Concentrated Liquid on Carbohydrate Metabolism. Jpn Pharmacol Ther 2006, 34:199-206.

4. Shizuma T, Ishiwata K, Nagano M, Mori H, Fukuyama N: Protective effects of fermented rice vinegar sediment (Kurozu moromimatsu) in a diethylnitrosamine-induced hepatocellular carcinoma animal model. J Clin Biochem Nutr 2011, 49:31-35.

5. Fukuyama N, Jujo S, Ito I, Shizuma T, Myojin K, Ishiwata K, Nagano M, et al.: Kurozu moromimatsu inhibits tumor growth of Lovo cells in a mouse model in vivo. Nutrition 2007, 23:81-86.

6. Shimoji Y, Kohno H, Nanda K, Nishikawa Y, Ohigashi H, Uenakai K, Tanaka T: Extract of Kurosu, a vinegar from unpolished rice, inhibits azoxymethane-induced colon carcinogenesis in male F344 rats. Nutr cancer 2004, 49:170-173.

7. Tanizawa H, Sazuka Y, Komatsu A, Takino Y: Acute toxicity of Kemezu and its effects on lipid metabolism in male mice. J Jpn Soc Nutr Food Sci 1983, 36:283-289. 
8. Hamadate N, Nakamura K, Hirai M, Yamamoto T, Yamaguchi H, Iizuka M, Yamamoto E, et al.: Effect of a dietary supplement containing Kurozu (a Japanese traditional health drink) concentrate on several obesity-related parameters in obese Japanese adults: a randomized, double-blind, placebo-controlled trial. Func Foods Health Dis 2013, 3:310-322.

9. Hamadate N, Seto K, Yazawa K: Effect of a dietary supplement containing Kurozu concentrate on body fat energy metabolism. Jpn J Complem Altern Med 2014, 11:67-74.

10. Abe S, Hasegawa M, Tsuruoka J, Matsumoto Y, Koyanagi S: Effect of dietary supplement containing Kurozu concentrate on visceral fat accumulation. Jpn J Complem Altern Med 2019, 16:13-19.

11. Vuppalanchi R, Chalasani N: Nonalcoholic fatty liver disease and nonalcoholic steatohepatitis: Selected practical issues in their evaluation and management. Hepatology 2009, 49:306-317.

12. Whitsett M, VanWagner LB: Physical activity as a treatment of non-alcoholic fatty liver disease: A systematic review. World J Hepatol 2015, 7:2041-52.

13. Preiss D, Sattar N: Non-alcoholic fatty liver disease: an overview of prevalence, diagnosis, pathogenesis and treatment considerations. Clin Sci (Lond) 2008, 115:141-150.

14. Younes R, Bugianesi E: Should we undertake surveillance for HCC in patients with NAFLD? J Hepatol 2018, 68:326-324.

15. Font-Burgada J, Sun B, Karin M: Obesity and Cancer: The Oil that Feeds the Flame. Cell Metab 2016, 23:48-62.

16. Ringelhan M, Pfister D, O'Connor T, Pikarsky E, Heikenwalder M: The immunology of hepatocellular carcinoma. Nat Immunol 2018, 19:222-232.

17. Wilson RA, Deasy W, Hayes A, Cooke MB: High fat diet and associated changes in the expression of micro-RNAs in tissue: Lessons learned from animal studies. Mol Nutr Food Res 2017, 61:1600943.

18. McGregor RA, Choi MS: microRNAs in the regulation of adipogenesis and obesity. Curr Mol Med 2011, 11:304-316.

19. Ortega FJ, Mercader JM, Catalan V, Moreno-Navarrete JM, Pueyo N, Sabater M, Gomez-Ambrosi J, et al.: Targeting the circulating microRNA signature of obesity. Clin Chem 2013, 59:781-792.

20. Murri M, El Azzouzi H: MicroRNAs as regulators of mitochondrial dysfunction and 
obesity. J Hepatol 2018, 68:326-324. Am J Physiol Heart Circ Physiol 2018, 315:H291-H302.

21. Icli B, Feinberg MW: MicroRNAs in dysfunctional adipose tissue: cardiovascular implications. Cardiovasc Res 2017, 113:1024-1034.

22. Dongiovanni P, Meroni M, Longo M, Fargion S, Fracanzani AL: miRNA Signature in NAFLD: A Turning Point for a Non-Invasive Diagnosis. Int J Mol Sci 2018, 19:3966.

23. Zarfeshani A, Ngo S, Sheppard AM: MicroRNA Expression Relating to Dietary-Induced Liver Steatosis and NASH. J Clin Med 2015, 4:1938-1950.

24. Lopez-Riera M, Conde I, Quintas G, Pedrola L, Zaragoza A, Perez-Rojas J, Salcedo M, et al.: Non-invasive prediction of NAFLD severity: a comprehensive, independent validation of previously postulated serum microRNA biomarkers. Sci Rep 2018, 8:10606.

25. Esquela-Kerscher A, Slack FJ: Oncomirs - microRNAs with a role in cancer. Nat Rev Cancer 2006, 6:259-269.

26. Slabakova E, Culig Z, RemSík J, Soucek K: Alternative mechanisms of miR-34a regulation in cancer. Cell Death Dis 2017, 8:e3100.

27. He Y, Yu D, Zhu L, Zhong S, Zhao J, Tang J: miR-149 in Human Cancer: A Systemic Review. J Cancer 2018, 9:375-388.

28. Pinkel D: The use of body surface area as a criterion of drug dosage in cancer chemotherapy. Cancer Res 1958, 18:853-856.

29. Kang K, Peng X, Luo J, Gou D: Identification of circulating miRNA biomarkers based on global quantitative real-time PCR profiling. J Anim Sci Biotechnol 2012, 3:4.

30. Wei J, Gao W, Zhu CJ, Liu YQ, Mei Z, Cheng T, Shu YQ: Identification of plasma microRNA-21 as a biomarker for early detection and chemosensitivity of non-small cell lung cancer. Chin J Cancer 2011, 6:407-414.

31. Tong LT, Katakura Y, Kawamura S, Baba S, Tanaka Y, Udono M, Kondo Y, et al.: Effects of Kurozu concentrated liquid on adipocyte size in rats. Lipids Health Dis. 2010, 9:134.

32. Fukuyama N, Shizuma T, Ishiwatari K, Nagano M: Effect of Kurozu ingestion on inflammatory bowel diseases in mice (Japanese). J Jpn Soc Parenteral Enteral Nutrition 2008, 23:S138.

33. Nanda K, Miyoshi N, Nakamura Y, Shimoji Y, Tamura Y, Nishikawa Y, Uenakai K, et al.: Extract of vinegar "Kurosu" from unpolished rice inhibits the proliferation of human cancer cells. J Exp Clin Cancer Res 2004, 23:69-75. 
34. Ishimoto T, Lanaspa MA, Rivard CJ, Roncal-Jimenez CA, Orlicky DJ, Cicerchi C, McMahan RH, et al.: High-fat and high-sucrose (western) diet induces steatohepatitis that is dependent on fructokinase. Hepatology 2013, 58:1632-1643.

35. Takakura K, Oikawa T, Nakano M, Saeki C, Torisu Y, Kajihara M, Saruta M: Recent Insights Into the Multiple Pathways Driving Non-alcoholic Steatohepatitis-Derived Hepatocellular Carcinoma. Front Oncol 2019, 9:762.

36. Duval C, Thissen U, Keshtkar S, Accart B, Stienstra R, Boekschoten MV, Roskams T, et al.: Adipose tissue dysfunction signals progression of hepatic steatosis towards nonalcoholic steatohepatitis in C57BL/6 mice. Diabetes 2010, 59:3181-3191.

37. Abdul-Wahed A, Guilmeau S, Postic C: Sweet Sixteenth for ChREBP: Established Roles and Future Goals. Cell Metab 2017, 26:324-341.

38. Kim HS, Ryoo ZY, Choi SU, Lee S: Gene expression profiles reveal effect of a high-fat diet on the development of white and brown adipose tissues. Gene 2015, 565:15-21.

39. Yan B, Guo Q, Fu FJ, Wang Z, Yin Z, Wei YB, Yang JR: The role of miR-29b in cancer: regulation, function, and signaling. Onco Targets Ther 2015, 8:539-548.

40. Jampoka K, Muangpaisarn P, Khongnomnan K, Treeprasertsuk S, Tangkijvanich P, Payungporn S: Serum miR-29a and miR-122 as Potential Biomarkers for Non-Alcoholic Fatty Liver Disease (NAFLD). Microrna 2018, 7:215-222.

41. Shimoji Y, Tamura Y, Nakamura Y, Nanda K, Nishidai S, Nishikawa Y, Ishihara N, et al.: Isolation and identification of DPPH radical scavenging compounds in Kurosu (Japanese unpolished rice vinegar). J Agric Food Chem 2002, 22:6501-6503.

42. Avtanski DB, Nagalingam A, Kuppusamy P, Bonner MY, Arbiser JL, Saxena NK, Sharma D: Honokiol abrogates leptin-induced tumor progression by inhibiting Wnt1-MTA1- $\beta$-catenin signaling axis in a microRNA-34a dependent manner. Oncotarget 2015, 18:16396-410.

43. Tsai WC, Hsu SD, Hsu CS, Lai TC, Chen SJ, Shen R, Huang Y, et al.: MicroRNA-122 plays a critical role in liver homeostasis and hepatocarcinogenesis. Cell Metab 2017, 26:324-341. J Clin Invest 2012, 122:2884-2897.

44. Deiuliis JA: MicroRNAs as regulators of metabolic disease: pathophysiologic significance and emerging role as biomarkers and therapeutics. Int J Obes (Lond) 2016, 40:88-101.

45. Shin CH, Lee H, Kim HR, Choi KH, Joung JG, Kim HH: Regulation of PLK1 through competition between hnRNPK, miR-149-3p and miR-193b-5p. Cell Death Differ 2017, 11:1861-1871. 
46. Okato A, Arai T, Yamada Y, Sugawara S, Koshizuka K, Fujimura L, Kurozumi A, et al.: Dual Strands of Pre-miR-149 Inhibit Cancer Cell Migration and Invasion through Targeting FOXM1 in Renal Cell Carcinoma. Int J Mol Sci 2017, 9: E1969.

47. Yang D, Du G, Xu A, Xi X, Li D: Expression of miR-149-3p inhibits proliferation, migration, and invasion of bladder cancer by targeting S100A4. Am J Cancer Res 2017, 11:2209-2219.

48. Feng X, Zhang C, Yang Y, Hou D, Zhu A: Role of miR-181a in the process of apoptosis of multiple malignant tumors: A literature review. Adv Clin Exp Med 2018, 27:263-270.

49. Yu DL, Zhang T, Wu K, Li Y, Wang J, Chen J, Li XQ, et al.: MicroRNA-448 suppresses metastasis of pancreatic ductal adenocarcinoma through targeting JAK1/STAT3 pathway. Oncol Rep 2017, 38:1075-1082.

50. Tang LY, Heller M, Meng Z, Yu LR, Tang Y, Zhou M, Zhang YE: Transforming Growth Factor- $\beta$ (TGF- $\beta$ ) Directly Activates the JAK1-STAT3 Axis to Induce Hepatic Fibrosis in Coordination with the SMAD Pathway. J Biol Chem 2017, 292:4302-4312.

51. Shi Y, Yang Z, Zhang T, Shen L, Li Y, Ding S: SIRT1-targeted miR-543 autophagy inhibition and epithelial-mesenchymal transition promotion in Helicobacter pylori CagA-associated gastric cancer. Cell Death Dis 2019, 10:625.

52. Mantilla-Escalante DC, Lopez de Las Hazas MC, Gil-Zamorano J, Del Pozo-Acebo L, Crespo MC, Martín-Hernández R, Del Saz A, et al.: Postprandial Circulating miRNAs in Response to a Dietary Fat Challenge. Nutrients 2019, 11:E1326. 